\title{
Coordinated Effect of Ascorbate Biosynthesis and Recycling in Maize Seed Germination and Seedling Establishment under Low Temperature
}

\author{
Senlin Xiao, Tianjun Xu, Yuandong Wang, Jinfeng Xing, Ronghuan Wang, Aiguo Su, Shuaishuai Wang, Wei Song * \\ and Jiuran Zhao * \\ Beijing Key Laboratory of Maize DNA Fingerprinting and Molecular Breeding, Maize Research Center, \\ Beijing Academy of Agriculture and Forestry Sciences, Beijing 100097, China; forestxiao@163.com (S.X.); \\ xtjxtjbb@163.com (T.X.); wyuandong@126.com (Y.W.); forestxiao@126.com (J.X.); ronghuanwang@126.com (R.W.); \\ sx_201.su@163.com (A.S.); yusan183@163.com (S.W.) \\ * Correspondence: songwei1007@126.com (W.S.); maizezhao@126.com (J.Z.)
}

Citation: Xiao, S.; Xu, T.; Wang, Y.; Xing, J.; Wang, R.; Su, A.; Wang, S.; Song, W.; Zhao, J. Coordinated Effect of Ascorbate Biosynthesis and Recycling in Maize Seed Germination and Seedling Establishment under Low Temperature. Agriculture 2021, 11, 1160. https://doi.org/ 10.3390 /agriculture 11111160

Academic Editors: Mumtaz Cheema, Mercè Llugany, Peter A. Roussos and Pirjo Mäkelä

Received: 26 October 2021

Accepted: 17 November 2021

Published: 18 November 2021

Publisher's Note: MDPI stays neutral with regard to jurisdictional claims in published maps and institutional affiliations.

Copyright: (C) 2021 by the authors Licensee MDPI, Basel, Switzerland. This article is an open access article distributed under the terms and conditions of the Creative Commons Attribution (CC BY) license (https:// creativecommons.org/licenses/by/ $4.0 /)$.

\begin{abstract}
The impacts of low temperature occasionally encountered at higher latitude regions on maize seed germination present significant threats to yield and cultivation. Exploring the association of antioxidant system with low temperature (LT) germination could support the breeding strategies for better responding to LT disturbance. In this study, we have examined the germination rate and growth potential of a set of elite maize inbred accessions under LT and normal temperature (NT) conditions in the field. These accessions were found to have variable germination rate and growth potential when grown at LT, whereas the difference is not significant under NT. Physiological study revealed lower hydrogen peroxide content in LT tolerant accessions when compared with sensitive ones. LT-tolerant and LT-sensitive lines maintained similar content of ascorbate (AsA) and glutathione (GSH), whereas the reduced substrate content of which were significantly higher in LT-tolerant accessions. Consistently, activities of ascorbate peroxidase and dehydroascorbate reductase, the enzyme components that responsible for the AsA-GSH recycling, were much higher in LT-tolerant lines. Transcription profile revealed the increased expression of ZmVTC2 gene in LT-tolerant inbred line, which was rate limited step in AsA biosynthesis. These data indicates that the coordinated improvement of AsA biosynthesis and AsA-GSH recycling increase the pool size of the total antioxidants, which ameliorate LT-induced oxidative stress during maize seed germination.
\end{abstract}

Keywords: maize; low-temperature germination; ascorbate

\section{Introduction}

Maize (Zea mays L.) is one of the most important agricultural crops with the largest yield and acreage in the world. Since maize originated from tropical and subtropical regions, the plant is sensitive to low temperature (LT), mainly during germination and post-germinative growth period [1,2]. The agricultural praxis is to sow maize when the soil temperature is approximately above $10{ }^{\circ} \mathrm{C}$. Under condition of soil temperature below $10{ }^{\circ} \mathrm{C}$, seed germination and post-germinative growth are significantly inhibited, that result to the reduction of crop yield [3,4]. In addition, periods of LT occasionally encountered at higher latitudes regions significantly threaten maize cultivation. Therefore, selection of elite maize inbred accessions should take into account the seed germination and postgerminative growth potential under LT stress.

Low temperature impaired plant stomatal function, photosystem activity, Calvin cycle, or photosynthetic enzyme activities, as well as altered electron transport chain reactions, which finally leading to the excessive accumulation of reactive oxygen species (ROS) and subsequently oxidative damage [5,6]. On the other hand, the generation of ROS is a fundamental process in higher plants and function to transmit signal response to the 
changing environment. Thus, plant developed a suit of antioxidant defense systems to maintain the equilibrium between generation and scavenging of ROS [6,7]. In general, the antioxidant defense system consists of nonenzymatic antioxidants and some antioxidant enzymes, both of them participate in the redox reaction by electron transfer to eliminate the oxidative damage. The enzyme superoxide dismutase (SOD) functions in the first line of ROS defense, converting superoxide $\left(\mathrm{O}_{2}{ }^{\bullet-}\right)$ into hydrogen peroxide $\left(\mathrm{H}_{2} \mathrm{O}_{2}\right)$. The generated $\mathrm{H}_{2} \mathrm{O}_{2}$ can be further converted into $\mathrm{H}_{2} \mathrm{O}$ by the enzymes catalase (CAT), ascorbate peroxidase (APX), glutathione peroxidase (GPX), or by the ascorbate-glutathione pathway (AsA-GSH pathway).

AsA-GSH pathway, also known as Asada-Halliwell pathway, is the major pathway that detoxifies the $\mathrm{H}_{2} \mathrm{O}_{2}$ in a plant cell [8]. This pathway comprises two nonenzymatic antioxidants, AsA and GSH, and four antioxidant enzymes, including APX, GR, monodehydroascorbate reductase (MDHAR), and dehydroascorbate reductase (DHAR). AsA and GSH both have high redox potentials; the maintenance of their redox state is important in conferring stress tolerance in plants. Redox state of AsA and GSH mainly depends on the activities of the four enzymes that catalyze the conversion between their redox and oxidative form. First, the enzyme APX converts $\mathrm{H}_{2} \mathrm{O}_{2}$ into the water with the help of AsA as an electron donor. The produced monodehydroascorbate (MDHA) recycled back to AsA by ferredoxin at photosystem I (PSI) and by MDHAR, or spontaneously converted into dehydroascorbate (DHA). DHA is then recycled to AsA by the activity of GSH-dependent DHAR. In this reaction, one electron is transferred from GSH to DHA, and GSH is oxidized to oxidized glutathione (GSSG) that is further reduced back to GSH by NADPH-dependent GR activity [6,8-10].

AsA and GSH function as the electron carrier and play pivotal role in ROS detoxification, the ratio of AsA/DHA and GSH/GSSG are considered as oxidative stress sensor for the surveillance of cellular redox homeostasis [11]. Plants activate the AsA-GSH pathway to cope with LT stress. For example, LT stress increased GSH/GSSG and AsA/DHA ratios in Citrullus lanatus [12]. Similarly, rice seedlings exposed to LT stress had enhanced GSH/GSSG ratio and along with increased SOD and CAT activity [13]. Antioxidants enzymes play key role in plant fitness to LT stress. For example, overexpression of $A P X$ gene from Camellia azalea improves ROS scavenging and cold tolerances in tobacco [14]. Furthermore, transgenic tobacco plants expressing a human DHAR gene showed enhanced tolerance to low temperature $\left(15^{\circ} \mathrm{C}\right)$ compared to non-transgenic plants [15].

Positive effect of AsA and GSH in stress tolerance has been validated in plenty of previous studies (reviewed by [8]). Nevertheless, DHA and GSSG function as oxidative substrate in AsA and GSH recycling, the effect of their content variation on LT stress tolerance is largely unknown. This study investigates the germination, post-germinative growth, as well as the antioxidant defense system in a set of elite maize inbred accessions under LT stress in the field.

\section{Materials and Methods}

\subsection{Plant Material and Germination Assay}

Experimental materials included Jing724, Jing72464, Jing464, JingX201, JingB547, and JingX005, which were all female parents of elite hybrid in China. Among them, Jing724 was the female parent of Jingke968, which was the second largest planted hybrid in China, reaching about one million of hectares until 2021. All these accessions were developed by the Maize Research Center of Beijing Academy of Agriculture and Forestry Sciences. Seeds used for germination analysis were prepared in Hainan province at the winter of 2020. Germination assays were conducted in Beijing farms $\left(40.1^{\circ} \mathrm{N}, 116.4^{\circ} \mathrm{E}\right)$ at 25 March and 7 June. From the beginning of sowing on March 25 to the end of the survey on April 13, the average temperature was $14{ }^{\circ} \mathrm{C}$, which represented low temperature (LT). From the beginning of sowing on 7 June to the end of the survey on 13 June, the average temperature was $28^{\circ} \mathrm{C}$, which represented normal temperature (NT). Daily temperature is recorded based on the public meteorological data of the experimental location, detail information 
about the daily temperature is shown in Table S1. Three plots were designed in breeding fields for three independent replicates. In each plot, 20 rows of each accession were planted with $50 \mathrm{~cm}$ interspace for each row in the horizontal direction. Each row contained 19 spots in the vertical direction, 3 seeds were carefully planted in each spot with $30-\mathrm{cm}$ interspace. Thus, each replicate for one accession included 1140 seeds. The number of germinated seeds was counted at 20 day (for LT) and 10 day (for NT) after planting. The emergence of coleoptile from the soil was considered as a sign of successful germination of seed. The germination rates were calculated as the percentage of germinated seeds versus the total seed number. Seeds used in germination assays were manual selected and divided into two parts for LT and NT germination assays, respectively. For post-germinative growth analysis, seedling heights were measured with 20 random plants of each material in one plot. The corresponding seedlings were collected to measure the fresh weight.

\subsection{Measurement of Chlorophyll Content}

The portable chlorophyll meter (SPAD-502, Minolta, Japan) was used to measure chlorophyll content. In this case, 10 seedlings were selected randomly at both LT and NT to measure chlorophyll content of the first true leaf. Three readings were obtained for each leaf, avoiding main veins during measurements.

\subsection{Quantification of $\mathrm{H}_{2} \mathrm{O}_{2}$ Content}

Total seedlings above the ground ( $0.5 \mathrm{~g}$ fresh weight) were homogenized in an ice bath with $5 \mathrm{~mL}$ of $3 \%(w / v)$ trichloroacetic acid. The homogenate was centrifuged at $12,000 \times g$ for $15 \mathrm{~min}$, and $0.2 \mathrm{~mL}$ of supernatant was added to $1 \mathrm{~mL}$ of FOX reagent $(110 \mathrm{mM}$ perchloric acid, $150 \mu \mathrm{M}$ xylenol orange, $2 \mathrm{mM}$ ferrous sulfate in 9:1 methanol:water). The mixture was incubated at room temperature for $15 \mathrm{~min}$. The absorbance was measured at the wavelength of $560 \mathrm{~nm}$ using a disposable plastic cuvette and the concentration was calculated using a standard curve. For the standard curve preparation, $0.2 \mathrm{~mL}$ of $\mathrm{H}_{2} \mathrm{O}_{2}$ at different concentrations was added into $1 \mathrm{~mL}$ of FOX reagent. The results were expressed as $\mu \mathrm{mol} \mathrm{g}{ }^{-1}$ fresh weight.

\subsection{Quantification of Superoxide Content}

The $\mathrm{O}_{2}{ }^{\bullet-}$ producing rate was measured based on the following method. Total seedlings $(0.2 \mathrm{~g})$ were homogenized in $2 \mathrm{~mL}$ potassium phosphate buffer $(50 \mathrm{mM} / \mathrm{L}, \mathrm{pH} 7.8)$ and centrifuged at $12,000 \times \mathrm{g} \mathrm{rpm}$ for $10 \mathrm{~min}$ at $4{ }^{\circ} \mathrm{C}$. The $0.2 \mathrm{~mL}$ supernatant was taken and mixed with incubation solution $(0.2 \mathrm{~mL}$ potassium phosphate buffer $(50 \mathrm{mM} / \mathrm{L}, \mathrm{pH} 7.8)$, $0.2 \mathrm{~mL}$ hydroxylamine hydrochloride $(10 \mathrm{mM} / \mathrm{L}))$. After incubated at $25^{\circ} \mathrm{C}$ for $1 \mathrm{~h}$, the mixture was mixed with $0.2 \mathrm{~mL}$ sulfanilic acid $(17 \mathrm{mM} / \mathrm{L})$ and $0.2 \mathrm{~mL} \alpha$-naphthylamine $(7 \mathrm{mM} / \mathrm{L})$ for $20 \mathrm{~min}$. Then the absorbance of the reaction solution was measured at $530 \mathrm{~nm}$. The standard curve with $\mathrm{NaNO}_{2}$ was used to calculate the production rate of $\mathrm{O}_{2}{ }^{\bullet}$. The results were expressed as nmol $\mathrm{g}^{-1}$ fresh weight.

\subsection{Measurement of Nonenzymatic Antioxidants Content}

Ascorbic acid (AsA) and the dehydroascorbic acid (DHA) content were measured using the commercially available kits (Solarbio, BC1230 and BC1240, Beiing, China). Glutathione (GSH) and oxidized glutathione (GSSG) content were measured using the commercially available kits (Solarbio BC1170 and BC1185, China). The AsA and GSH redox state were calculated by the ratio of reduced and oxidized AsA and GSH, respectively. Proline was determined spectrophotometrically using the commercially available kits (Solarbio, BC0290, Beiing, China).

\subsection{Measurement of Activities of Antioxidant Enzymes}

For activity of antioxidant enzymes, total seedlings ( $0.5 \mathrm{~g}$ fresh weight) were homogenized in $5 \mathrm{~mL}$ phosphate buffer at $4{ }^{\circ} \mathrm{C}(1 \%$ polyvinylpyrrolidone, $1 \mathrm{mM}$ EDTA, and 
$5 \mathrm{mM}$ AsA, pH7.0). The homogenate was centrifuged at $12,000 \times \mathrm{g}$ rpm for $25 \mathrm{~min}$, and the supernatant was used as the source of enzymes for measurement.

Superoxide dismutase (SOD) activity was measured using a Total Superoxide Dismutase Assay Kit (Beyotime, Shanghai, China) based on the ability of SOD to inhibit the reduction of WST-8 formazan dye by the xanthine oxidase/xanthine reaction. One SOD enzymatic activity unit was defined as the amount of sample needed to achieve $50 \%$ inhibition of the rate of WST- 8 formazan dye reduction. The concentration of cellular total protein was quantified via Enhanced BCA Protein Assay Kit (Beyotime, P0012S, Beiing, China). The SOD activities were the ratio of total enzyme activity unit to the total protein, and the results were expressed as $\mathrm{U} / \mathrm{mg}^{-1}$ protein.

The APX activity was measured according to the manufacturer's instructions (Solarbio BC0020, Beiing, China). Supernatant containing APX enzyme were added to the the reaction mixture (50 mM potassium phosphate buffer (pH7.0), $0.1 \mathrm{mM}$ EDTA, $0.2 \mathrm{mM}$ $\mathrm{H} 2 \mathrm{O} 2,15 \mathrm{mM}$ AsA). The reaction was carried out when AsA was added. The result was calculated using the change of the absorbance at $290 \mathrm{~nm}$ after $5 \mathrm{~min}$. The results were expressed as nmol $\mathrm{min}^{-1} \mathrm{mg}^{-1}$ protein.

The DHAR activity was determined using a commercially available kit from Cominbio company (Cominbio, Jiangsu, China). DHAR activity was determined by monitoring the increase in absorbance at $265 \mathrm{~nm}$ owing to AsA formation. The reaction was initiated by adding DHA and results were expressed as nmol min ${ }^{-1} \mathrm{mg}^{-1}$ protein.

MDHAR, GR, and CAT activities were measured using the commercially available kits (Solarbio, MDHAR-BC0560; GR-BC1160; and CAT-BC0200, Beiing, China).

\subsection{RNA Extraction and Expression Analysis}

Total seedlings at LT or NT were sampled from inbred Jing72464 and JingX201. Whole seedlings with the first expanded true leaf were scissored from the upper ground, wrapped in the foil paper, then were directly frozen in the portable liquid nitrogen container in the field. Total RNA was isolated using the Plant RNA Purification Kit (Thermo Fisher Scientific, K0801, Waltham, MA, USA). Full-length cDNA was generated from $2 \mu \mathrm{g}$ total RNA per sample using oligo(dT) primer (cDNA synthesis kit, Thermo Fisher Scientific, K1622). The resulting cDNA were made a 1:10 dilution for the following quantitative RT-PCR (qRT-PCR). qRT-PCR was conducted using TB Green ${ }^{\circledR}$ Premix Ex Taq ${ }^{\text {TM }}$ II RealTime PCR System (Takara, Japan, RR820A) in a typical $20 \mu \mathrm{L}$ PCR mixture that included $10 \mu \mathrm{L}$ of premix, $3 \mu \mathrm{L}$ of template cDNA, and $0.4 \mu \mathrm{M}$ of each PCR primer. Cycling conditions were $95^{\circ} \mathrm{C}$ for $2 \mathrm{~min}$, followed by 40 cycles at $95^{\circ} \mathrm{C}$ for $20 \mathrm{~s}, 56^{\circ} \mathrm{C}$ for $1 \mathrm{~min}$, and $72{ }^{\circ} \mathrm{C}$ for $30 \mathrm{~s}$, and samples were run on the LightCycler 480 Real-Time PCR System (Roche, Basel, Switzerland). The melt curve data were utilized in every experiment to verify that the appropriate PCR product was being amplified. ZmUBI2 was used as the internal control to monitor sample uniformity of initial RNA input and reverse transcription efficiency. The expression of the genes of interest under each experimental condition was calculated by using the standard curve method [16], while the $2^{-\Delta \Delta C t}$ method was used to calculate the expression. The relevant primer sequences are in Table S2.

\subsection{Statistical Methods}

The statistical analysis was performed using IBM SPSS Statistics software version 20 (IBM, New York, NY, USA). For multiple comparisons, significance analysis was performed with two-way ANOVA followed by Bonferroni test. Values are presented as means and SEM and differences with values $<0.05$ are considered significant. Detail analysis results are given in Table S3.

\section{Results}

3.1. Germination Rate of Different Elite Maize Inbred Accessions under Low Temperature

A set of six elite maize inbred accessions [17], including Jing724, Jing72464, Jing464, JingX201, JingB547, and JingX005, were selected to test the germination rate under low 
temperature (LT) in early spring and normal temperature (NT) in summer in the field condition. The emergence of coleoptile from the soil was evaluated as successful germination of seed. All accessions maintained a high germination rate at NT, almost all seeds germinated at 7-day after plating (Figure 1a). No obvious differences were observed among different germplasm (Figure 1b). Compared to the control, LT significantly suppressed seed germination. The first batch of coleoptile emerged from the soil at about 14 day after plating, which was obviously longer than that at NT (Figure 1a). Even by day 20 when the average temperature gradually going up to $19{ }^{\circ} \mathrm{C}$, seed germination rate remained below $60 \%$ in Jing72464, which performed best among the six accessions (Figure 1c). Germination rate varied extensively among six germplasms under LT condition, highly significant differences $(p<0.001)$ were observed among different inbred lines. Jing724, Jing72464 and Jing 464 had a relatively high germination rate of $\sim 65 \%$ at LT, while accessions JingX201 and JingB547 had a germination rate of $25 \%$ and $31 \%$, respectively. When evaluate using the inhibition rate of LT, Jing72464 were the most tolerant accession, while JingX201 was the most sensitive one (Figure 1c).

\subsection{Variation in Seedling Establishment among Maize Accessions under Low Temperature}

Low temperature also suppressed the seedling establishment of maize. Plant heights of the seedlings were calculated to compare the post-germinative growth potential in response to LT. Results indicated that there were significant differences between accessions under LT condition $(p<0.001)$. In general, seedling heights of inbred Jing724, Jing72464, and Jing 464 were $4.5 \mathrm{~cm}, 5.7 \mathrm{~cm}$, and $5.3 \mathrm{~cm}$, respectively, whereas seedling height of JingX201 and JingB547 were $2.7 \mathrm{~cm}$ and $3.9 \mathrm{~cm}$, respectively (Figure 2a,b). We chose accessions Jing72464 and JingX201 for further studies, which represent the LT-tolerant and LT-sensitive germplasm among the six accessions (Figure 2c). The seedling height of Jing72464 was obviously higher than that of JingX201 at LT, whereas there was no obvious difference between two germplasm at NT.

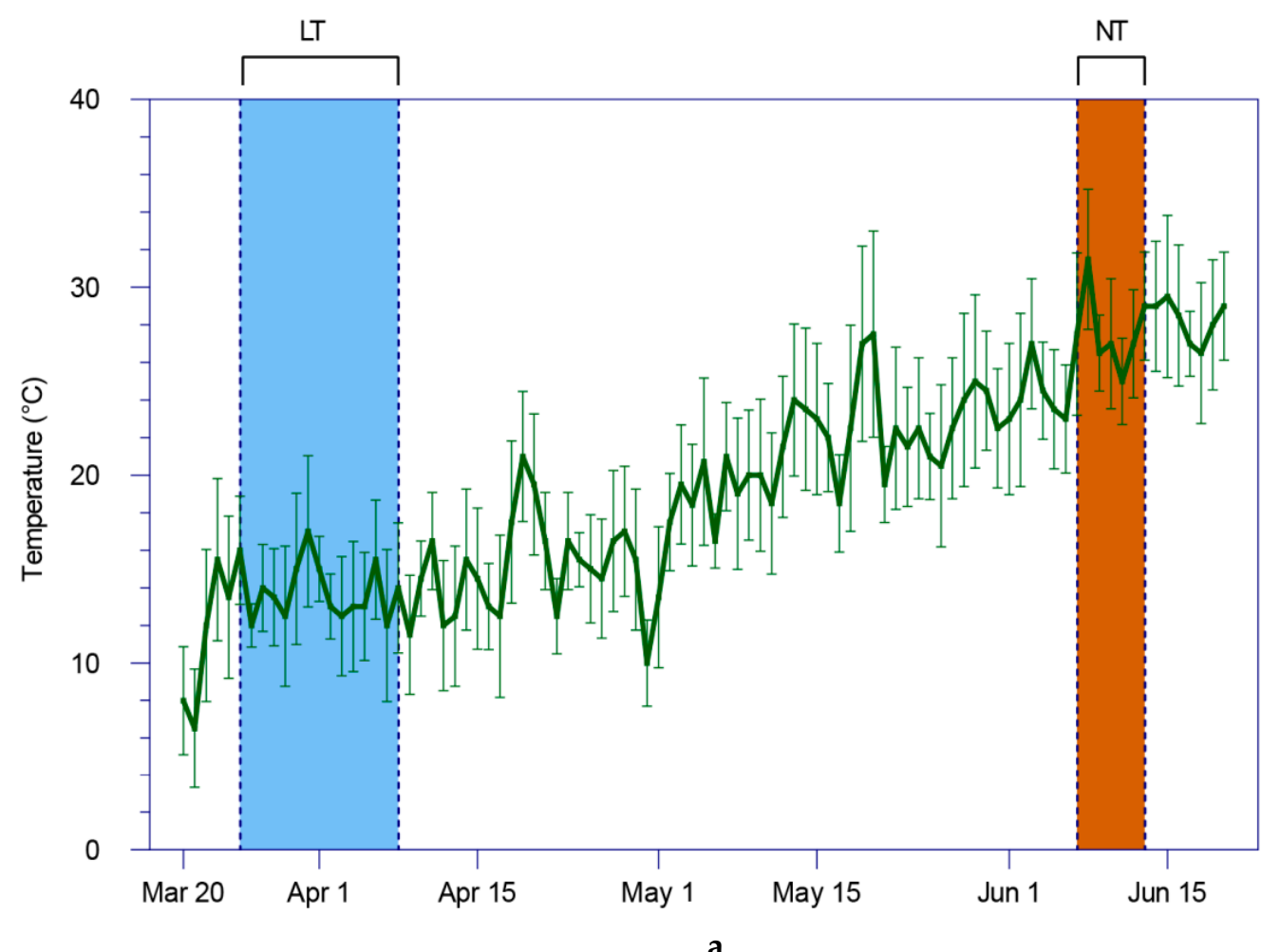

Figure 1. Cont. 

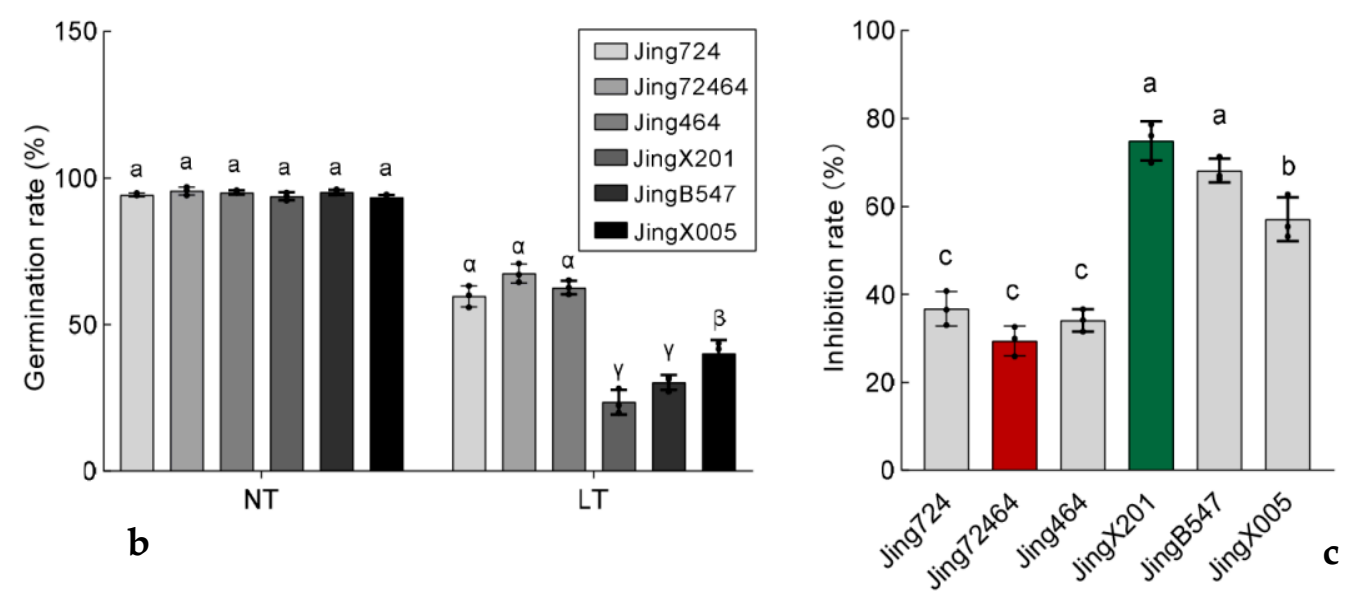

Figure 1. Germination rate of different elite maize inbred accessions under low temperature (a) Daily average temperature (green line) from 20 March to 20 June in the field where experiment conducted. The color-filled zone indicated the duration time after sowing when 5 percent coleoptile of Jing72464 emerged from the soil. Blue color zone indicated low temperature (LT) condition that seeds were sowing in early spring, while brown color zone indicated normal temperature (NT) condition that seeds were sowing in summer. (b) Germination rate of 6 maize inbred accessions in the filed condition under LT or NT. Data represent mean values of three independent replicate $(n>1000)$. (c) Inhibition rate of LT on seed germination. Inhibition rate was calculated as the germination rate in LT versus that in NT. Significant differences in $(\mathbf{b}, \mathbf{c})$ were labeled with different Roman $(a, b, c)$ or Greek letters $(\alpha, \beta, \gamma)$.

Furthermore, inbred Jing72464 already developed dark green coleoptile and expanded true leaf, whereas JingX201 had pale green coleoptile and retarded true leaf (Figure 2a). Data shown that Jing72464 had the highest chlorophyll content among six inbred, which was $\sim 13.5 \%$ higher than that of JingX201 (Figure 2d). Collectively, low temperature negatively regulates seed germination and seedling establishment of the maize, whereas different germplasm exhibited variable response to LT.

\subsection{Ameliorated Oxidative Stress in LT-Tolerant Accession}

Low temperature causes overproduction of ROS in maize through imbalanced ROS detoxification and degradation of membrane fluidity [18]. Cellular ROS contents were measured in inbred Jing72464 and JingX201. As an indicator of oxidative stress, $\mathrm{H}_{2} \mathrm{O}_{2}$ content in Jing72464 was $\sim 28 \%$ lower than that of JingX201 $(p<0.001)$ (Figure 3a). Nevertheless, $\mathrm{O}_{2}{ }^{\bullet-}$ contents were slightly higher in Jing72464 than JingX201 $(p=0.029)$ (Figure 3b). ROS can induce lipid peroxidation, which results in the production of malondialdehyde (MDA) [19]. Under LT condition, the MDA content in Jing72464 was 39\% lower than JingX201 $(p<0.001)$, indicating the ameliorated oxidative stress (Figure 3c). Plants tend to increase the content of soluble osmolytes, e.g., proline, to counteract the abiotic stress [20]. However, proline content in Jing72464 had no difference as that of JingX201 ( $p=0.325)$ (Figure 3d). In summary, the reduced accumulation of $\mathrm{H}_{2} \mathrm{O}_{2}$, together with the decreased MDA content in JingX201, represented the ameliorated cellular oxidative stress in LTtolerant inbred Jing72464.

\subsection{Coordinated Antioxidant Defense Systems in LT-Tolerant Accession}

Plant tends to ameliorate the cellular oxidative stress by promoting the activity of antioxidant defense system. We profiled activities of antioxidant defense systems in Jing72464 and JingX201 under NT and LT conditions. Activity of SOD shown no difference between two inbred lines $(p=0.946)$, while activity of CAT was slightly higher $(13.9 \%)$ in Jing72464 than that of JingX201 ( $p=0.013)$ (Figure S1). Among the four antioxidant enzymes in AsA-GSH pathway, activities of GR and MDHAR showed no difference between the 
two inbred lines (Figure S1). APX, functions as a key enzyme in the fine control of $\mathrm{H}_{2} \mathrm{O}_{2}$, showed 34\% higher activity in Jing72464 than that of JingX201 $(p<0.001)$ (Figure 4a). DHAR, function in the direction of DHA consuming with the help of GSH, showed $54 \%$ reduced activity in Jing72464 when compared with JingX201 $(p<0.001)$ (Figure 4b). Among the nonenzymatic antioxidants in AsA-GSH pathway, no difference in the AsA content was observed between Jing72464 and JingX201 $(p=0.298)$. However, DHA content was significantly higher $(53.6 \%)$ in Jing72464 than that of JingX201 $(p<0.001)$ (Figure $4 c, d)$. Consistently, the GSH content in Jing72464 was slightly higher (32\%) than that of JingX201, whereas GSSG (oxidized form of GSH) content was more than 1.2-fold higher (121.7\%) in Jing72464 than that of JingX201 $(p<0.001)$ (Figure 4f,g). This result implied that activities of APX and DHAR is not only associated with the antioxidants level of AsA and GSH, but also with the content of oxidative substrate of these two antioxidants. As the result, pool size of total ascorbate (AsA + DHA) was 23\% higher in LT-tolerant inbred Jing72464 than LT-sensitive inbred JingX201 (Figure 4e). Similarly, pool size of total glutathione (GSH + GSSG) was 38\% higher in Jing72464 than JingX201 $(p<0.001)$ (Figure 4h). The AsA/DHA and GSH/GSSG ratios were $40.8 \%$ and $40.6 \%$ lower in Jing72464, respectively (Figure $4 \mathrm{i}, \mathrm{j}$ ).

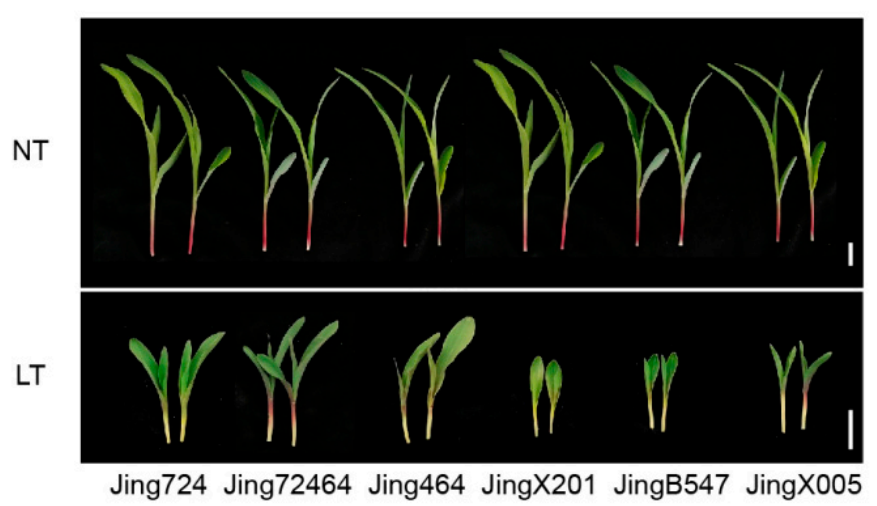

Jing724 Jing72464 Jing464 JingX201 JingB547 JingX005

a

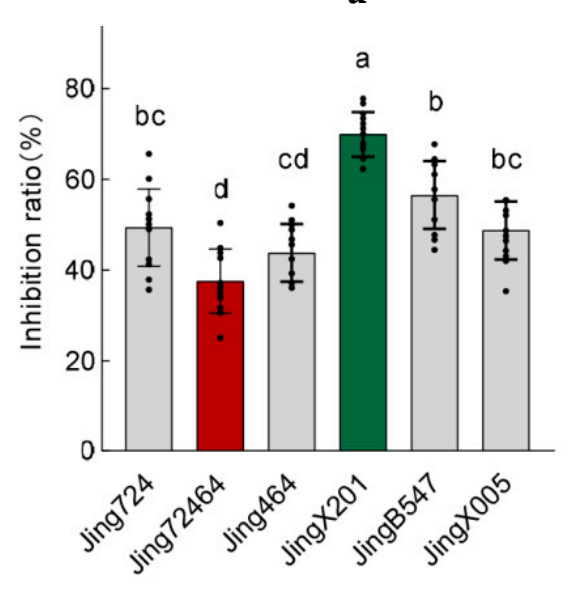

C

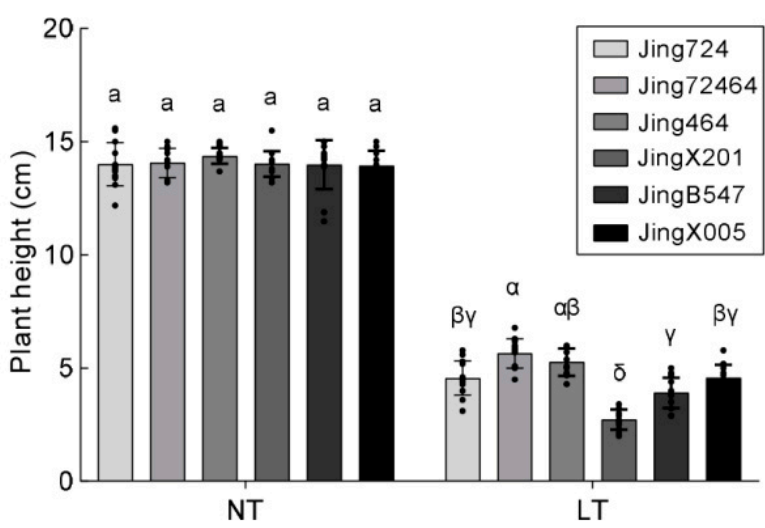

b

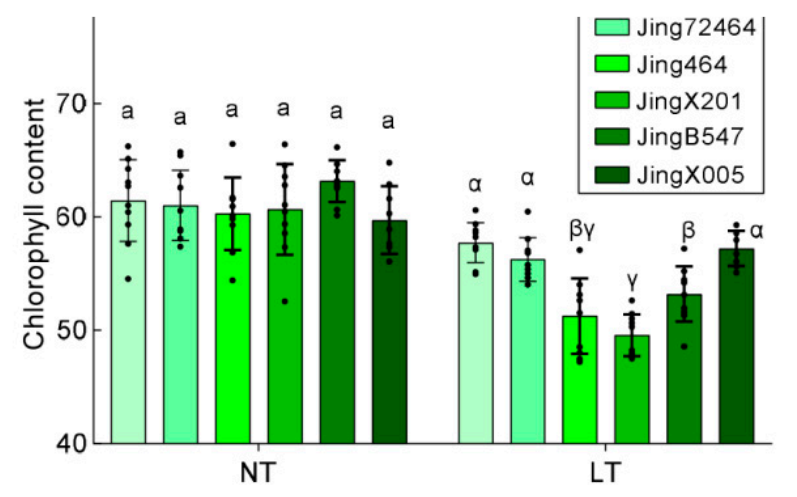

Figure 2. Variation in seedling establishment among maize accessions under low temperature. (a) Plant morphology of seedlings with the first true leaf expanded. Scale bar, $2 \mathrm{~cm}$. (b) Plant height of 6 maize inbred accessions in the filed condition under LT or NT. Data represent mean values of three independent replicate $(n>12)$. (c) Inhibition rate of LT on plant height. Data were calculated as the plant height in LT versus the means in NT. $(n>12)$. (d) Chlorophyll content of 6 maize inbred accessions in the filed condition under LT or NT. Data are mean \pm s.e.m. $(n=10)$. (b-d) Significant differences were labeled with different Roman $(a, b, c)$ or Greek letters $(\alpha, \beta, \gamma)$. 


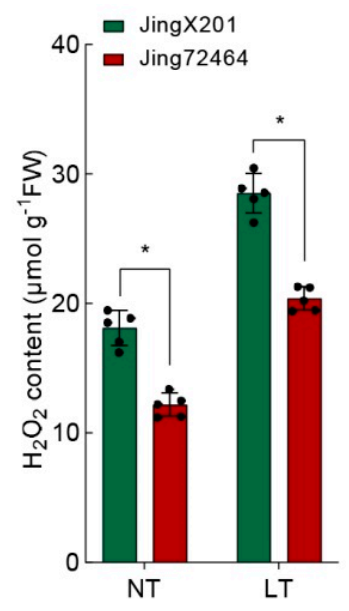

a

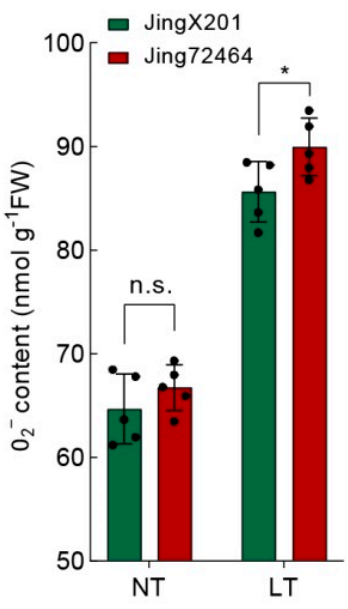

b

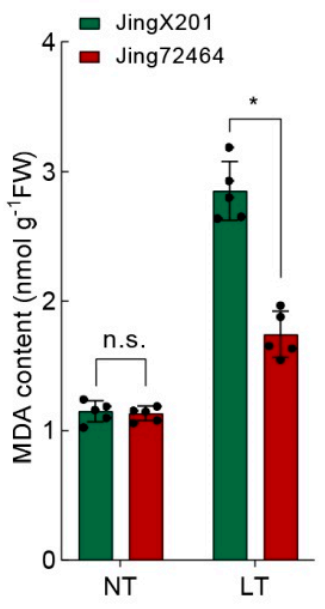

C

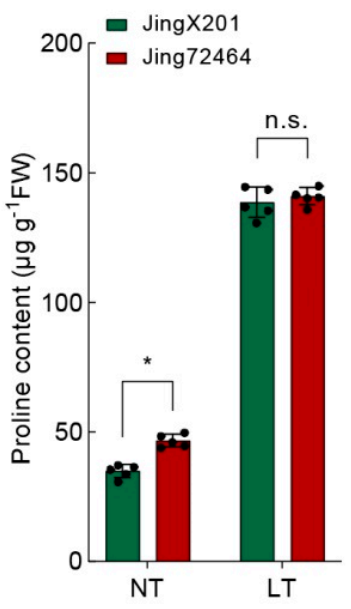

d

Figure 3. Ameliorated oxidative stress in LT-tolerant inbred accession. (a) Superoxide content in LT-sensitive inbred JingX201 and LT-tolerant inbred Jing72464 under NT and LT condition. (b) Hydrogen peroxide content. (c) MDA content. (d) Proline content. (a to d) Data are mean \pm s.e.m. $(n=5)$. n.s. means no significance, ${ }^{*}$ means $p<0.05$.

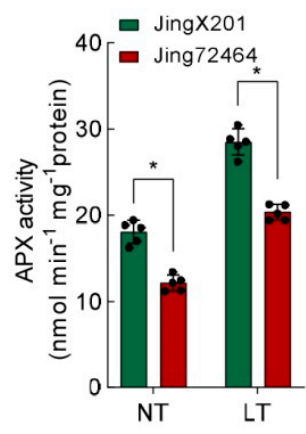

a

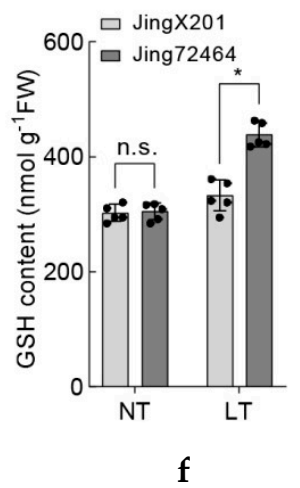

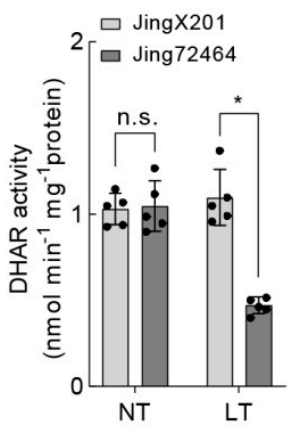

b

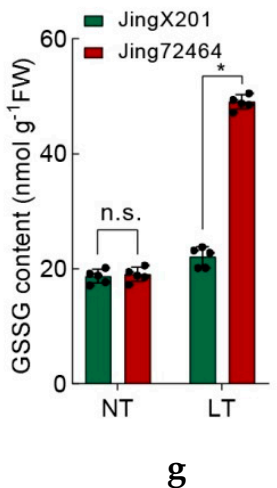

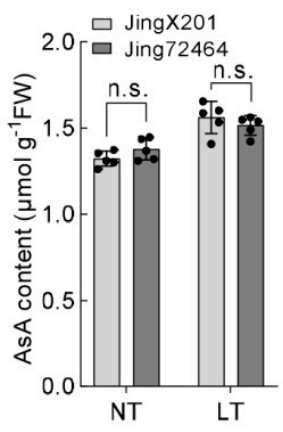

C

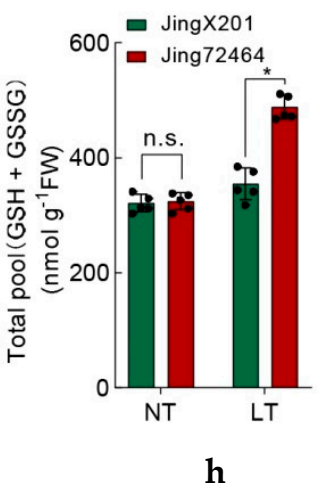

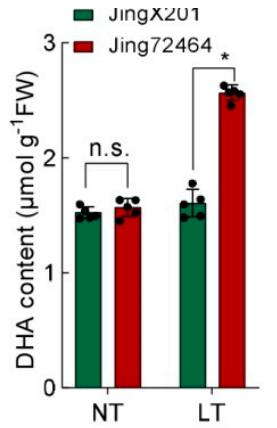

d

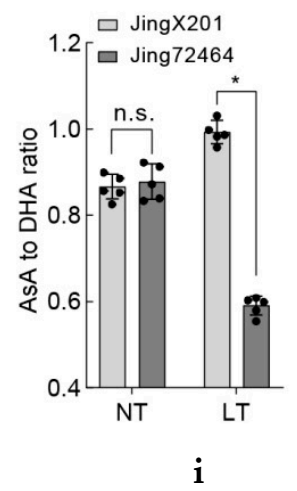

Figure 4. Enhanced GSH-AsA redox status in LT-tolerant accession Jing72464. (a,b) Cellular activity of ascorbate peroxidase (a) and dehydroascorbate reductase (b) in LT-sensitive inbred JingX201 and LT-tolerant inbred Jing72464 under NT and LT condition. (c,d) Cellular contents of AsA (c) and DHA (d). (e) Total AsA-DHA pool, caculated as the combination of AsA and DHA content. (f, $\mathbf{g})$ Cellular contents of GSH (f) and GSSG (g). (h) Total GSH-GSSG pool, caculated as the combination of GSH and GSSG content. (i,j) The ratio of AsA/DHA (i) and GSH/GSSG (j). Data are mean \pm s.e.m. $(n=5)$. n.s. means no significance, * means $p<0.05$. 


\subsection{Transcriptional Profile Analysis of AsA-GSH Pathway}

The nonenzymatic antioxidant pool of AsA-GSH pathway undergoes turnover along with the redox function metabolism in plants, therefore the biosynthesis, degradation, and recycling of these compound not only maintained the reduced/oxidized ratio, but also the total buffer pool [8]. We next set to explore the transcriptional variation between Jing72464 and JingX201. Gene expression in biosynthesis, degradation, and recycling of AsA-GSH pathway was detected using semi-quantitative RT-PCR. AsA is produced via the D-mannose/L-galactose pathway, in which GGP catalyzes the conversion of GDP-Lgalactose to L-galactose 1-P and is the first committed step in this pathway [21]. Transcription level of ZmVTC2, GGP encoding gene in maize, was significantly increased in response to LT stress. At NT condition, transcripts level of ZmVTC2 in Jing72464 was about 4.3-fold higher in Jing72464 than JingX201 $(p<0.001)$, indicating the enhanced production of AsA pool. Other genes encoding catalyzing enzymes of this pathway, including ZmGME, ZmVTC4, ZmGalDH, and ZmGalLDH were not significantly changed between two genotypes (Figure S2). Transcription level of GSH biosynthesis genes, including ZmECS, ZmGSH1, and ZmGSH2 were induced at different degree in response to LT, while there was no significant difference between the two inbred lines (Figure S2). Transcript levels of genes encoding enzymes in recycling of AsA-GSH pathway were analyzed. Among the four antioxidant enzymes in AsA-GSH recycle pathway, major difference came from $\mathrm{ZmAPX}$, which use AsA as an electron donor to detoxify $\mathrm{H}_{2} \mathrm{O}_{2}$. Transcription level of ZmAPX1 and ZmAPX2 were significantly higher in Jing72464 than that of JingX201 $(p<0.001)$ (Figure 5a). Furthermore, ascorbate oxidase (AO) catalyzes the oxidation of AsA to MDHA, with the concomitant reduction of molecular oxygen to water. Transcription level of ZmAO was 4.1-fold higher in Jing72464 than that of JingX201 $(p<0.001)$ (Figure 5a). The other three antioxidant enzymes in AsA-GSH recycle pathway, including ZmMDHAR, ZmDHAR, and ZmGR, also increased transcription in response to LT, but no significant differences were observed between two genotypes (Figure S2).

Transcription of SOD, CAT, and GST were also analyzed. Majority of the analyzed genes increased in response to LT. For example, transcripts levels of ZmSODA.3, ZmSODB, and ZmGST2 were much higher in Jing72464 than JingX201 under LT condition, whereas transcripts level of ZmSOD4 was lower in Jing72464 (Figure S2). Collectively, our results suggested that enhancement of the GSH and AsA redox pools improved LT tolerance of maize seedlings, which might be conferred by the coordination of VTC2-mediate AsA biosynthesis and APX-mediate antioxidants recycling (Figure 5a).

The ratios of AsA to DHA and GSH to GSSG may signal the plant with appropriate response to abiotic stress, which coordinate the actions of multiple signaling pathways in regulation of plant development. As illustrated in Figure 2, LT-tolerant inbred Jing72464 maintain the considerable post-germinative growth and normal photosynthesis. Compared with that, LT-sensitive inbred JingX201 had strong response to LT stress, leading to the impairment of seedling establishment and photosynthesis in subsequent growth. Consequently, Jing72464 maintained considerable plant height, biomass, and production of seeds at mature stage, whereas JingX201 was significantly suppressed on these physiological indexes (Figures 6 and S3). 

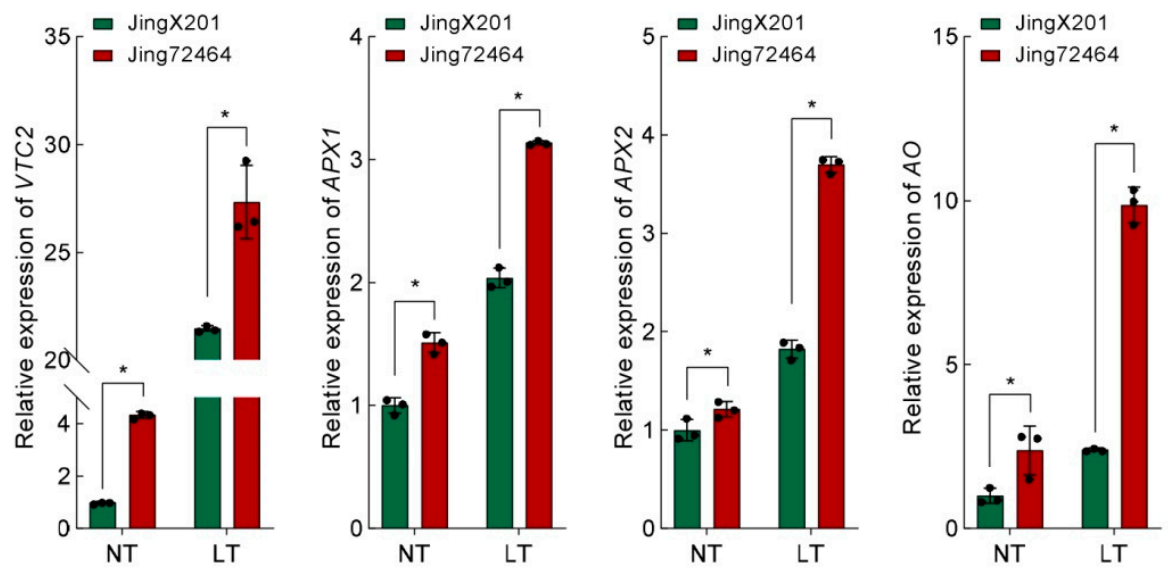

a
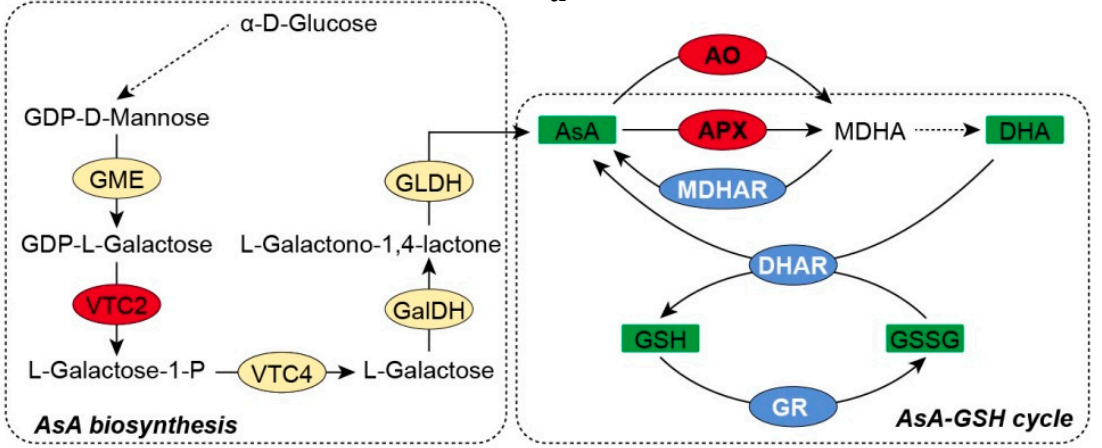

b

C

Figure 5. Transcription profile revealed the enhanced AsA biosynthesis and recycling in LT-tolerant inbred Jing72464. $(\mathbf{a}, \mathbf{b})$ Transcript levels of key enzymatic components in AsA biosynthesis (a) and AsA-GSH recycle pathway (b) in Jing72464 and JingX201. Transcription is measured relative to that of JingX201 seedlings under NT condition (set to 1). Data are mean \pm s.e.m. $(n=3)$, n.s. means no significance, ${ }^{*}$ means $p<0.05$. (c) Schematic representation of AsA biosynthesis and AsA-GSH recycle pathway.

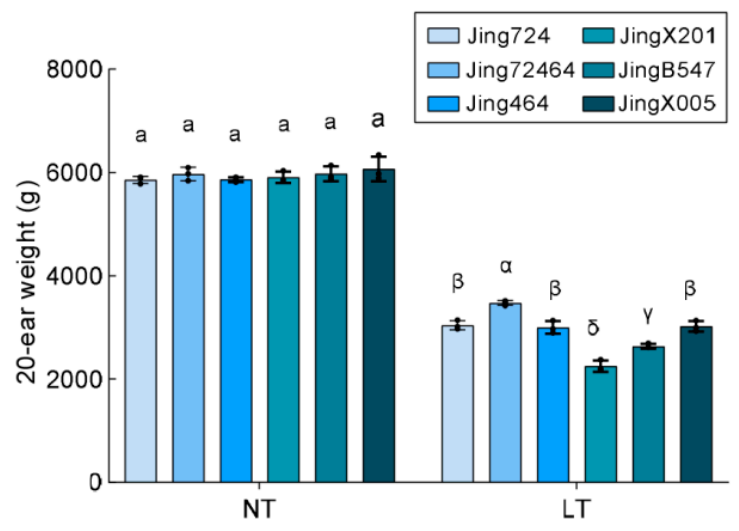

a

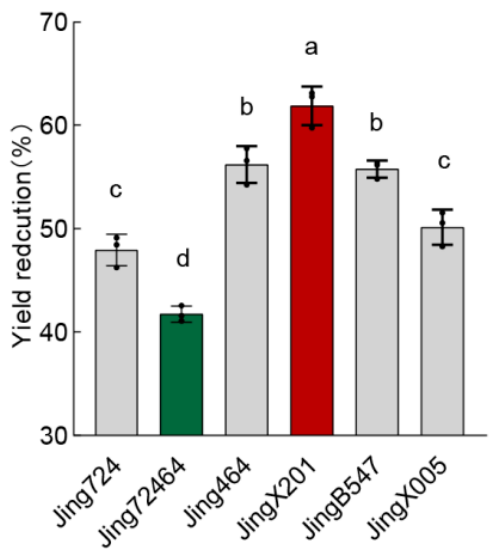

b

Figure 6. Yield of Jing72464 was less inhibited by low temperature. (a) Yield of 6 maize inbred accession in the filed condition under LT or NT. Data represent mean values of three independent replicate $(n>10)$. Significant differences were labeled with different Roman $(a, b, c)$ or Greek letters $(\alpha, \beta, \gamma)$. (b) Yield reduction ratio was calculated as the yield in LT versus that in NT. 


\section{Discussion}

To counteract the oxidative damage that continuously occurred due to abiotic stress, plant developed multiple enzymatic and non-enzymatic antioxidative defense systems to maintain the redox balance in the cell. The AsA-GSH pathway plays a vital role in detoxifying ROS and interacts with other defense systems to counteract various abiotic stresses. Both AsA and GSH are strong antioxidants which can donate one or two electrons to form oxidized form, DHA and GSSG, respectively. AsA is of particular importance among these, and is the most abundant water-soluble antioxidant in plants, e.g., AsA is present at concentrations of $20 \mathrm{mM}$ or more in chloroplasts. In addition, AsA serves as an important co-factor in several pathways including the xanthophyll cycle, the synthesis of ethylene, and cell wall components. AsA is also involved in the regulation of cell division and cell expansion. Thus, the maintenance of proper AsA content is essential to the plant development and abiotic stress tolerance [6,8,21-23].

Recycling of AsA from their oxidized forms is the necessity to keep the redox balance as well as the higher AsA content. The oxidized ascorbate, MDHA or DHA, can be recycled back to its reduced form by MDHAR or DHAR, respectively. Since MDHAR and DHAR are not rate-limiting steps in the recycling of AsA, loss-of-function mutations of their coding genes did not affect the pool size of total ascorbate [24,25]. In this study, LT-tolerant inbred Jing72464 maintained similar AsA and GSH content as that of sensitive ones, whereas the oxidized substrates were significantly higher. This led to the increase of pool size in total ascorbate and glutathione, which contribute to the stress fitness of plant. Among the four antioxidative enzymes in AsA-GSH pathway, ZmAPX1 and ZmAPX2 showed significant transcriptional differences between the LT-tolerant and LT-sensitive inbred lines. Our results are consistent with previous study, in which APX gene was more inducted by LT in LT-tolerant maize genotype Gurez local but not in LT-susceptible genotype G-M-6 [26]. This implied that APX might be the key enzyme to regulate low temperature tolerance of maize.

Plant activates the AsA-GSH recycle pathway to ameliorate the oxidative damage, meanwhile requires the fine coordination of de novo antioxidant biosynthesis and recycling. There is compelling genetic evidence that the biosynthesis of AsA proceeds via the D-mannose/L-galactose pathway and is the most significant source of ascorbate in plants. This pathway consists of eight reaction processes catalyzed by constitutive enzymes, including GME, GGP, GPP, L-GalDH, L-GalLDH, and so on. GGP/VTC2 is the rate-limiting step in this pathway and is the only gene that causes a substantial increase in ascorbate contents by its overexpression [21,27]. Among the biosynthesis genes analyzed in these studies, ZmVTC2 is the only gene that significantly induction by LT, which suggested its predominant role for abiotic stress fitness of maize. LT-tolerant inbred Jing72464 maintained significantly higher transcription level of ZmVTC2 at normal condition than that of LT-sensitive inbred JingX201, which might provide the plant with higher AsA pool to counteract the oxidative damage due to abiotic stress perturbation, e.g., occasional encountered low temperature during germination. In this context, ZmVTC2 would be a major target of genetic engineering for the improvement of AsA content and LT-stress fitness.

\section{Conclusions}

We have here shown the association of total AsA-GSH pool with the low temperature stress tolerance in maize germination and post-germinative growth. The elite maize inbred Jing72464 will be used as LT-resistant maize germplasm and resource reserves to overcome the problem of low seed germination rate in Northeast China. The future research on AsA-GSH pathway under other stress condition would reveal additional important targets and improve our understanding of how the plant coordinate the de novo AsA biosynthesis with recycling to maintain the redox homeostasis in plant. 
Supplementary Materials: The following are available online at https:/ / www.mdpi.com/article/10 .3390 /agriculture11111160/s1, Figure S1: Cellular activity of key antioxidative enzymes in Jing72464 and JingX201, Figure S2: Transcription profile of key components in antioxidative system, Figure S3: Physiological analysis of maize inbred at mature stage. Table S1: Daily temperature during germinative experiment, Table S2: The primer sequences used for qRT-PCR assays, Table S3: two-way ANOVA analysis result.

Author Contributions: Conceptualization, S.X., W.S. and J.Z.; methodology, S.X. and T.X.; validation, A.S. and S.W.; formal analysis, S.X.; investigation, J.X.; resources, Y.W. and R.W.; writing-original draft preparation, S.X.; writing-review and editing, S.X. and W.S.; visualization, S.X. and W.S.; supervision, W.S. and J.Z.; funding acquisition, S.X. All authors have read and agreed to the published version of the manuscript.

Funding: This research was funded by Youth Research Fund (QNJJ202128) and Innovation Capability Construction Special Project (KJCX20180413) of Beijing Academy of Agriculture and Forestry Sciences, This work was also supported by the Beijing Scholars Program (BSP041), Science and Technology Xing Meng action foucus project of Inner Mongolia Autonomous Region (KJXM2020001).

Institutional Review Board Statement: Not applicable.

Informed Consent Statement: Not applicable.

Data Availability Statement: Data is contained within the article and supplementary material.

Acknowledgments: We fully appreciate the editors and all anonymous reviewers for their constructive comments on this manuscript.

Conflicts of Interest: The authors declare no conflict of interest. The funders had no role in the design of the study; in the collection, analyses, or interpretation of data; in the writing of the manuscript, or in the decision to publish the results.

\section{References}

1. yyGreaves, J.A. Improving suboptimal temperature tolerance in maize-the search for variation. J. Exp. Bot. 1996, 47, 307-323. [CrossRef]

2. Xue, X.; Du, S.; Jiao, F.; Xi, M.; Wang, A.; Xu, H.; Jiao, Q.; Zhang, X.; Jiang, H.; Chen, J.; et al. The regulatory network behind maize seed germination: Effects of temperature, water, phytohormones, and nutrients. Crop. J. 2021, 9, 718-724. [CrossRef]

3. Verheul, M.J.; Picatto, C.; Stamp, P. Growth and development of maize (Zea mays L.) seedlings under chilling conditions in the field. Eur. J. Agron. 1996, 5, 31-43. [CrossRef]

4. Li, X.; Wang, G.; Fu, J.; Li, L.; Jia, G.; Ren, L.; Lubberstedt, T.; Wang, G.; Wang, J.; Gu, R. QTL Mapping in Three Connected Populations Reveals a Set of Consensus Genomic Regions for Low Temperature Germination Ability in Zea mays L. Front. Plant Sci. 2018, 9, 65. [CrossRef]

5. Dreyer, A.; Dietz, K.J. Reactive Oxygen Species and the Redox-Regulatory Network in Cold Stress Acclimation. Antioxidants 2018, 7, 169. [CrossRef]

6. Hasanuzzaman, M.; Bhuyan, M.; Zulfiqar, F.; Raza, A.; Mohsin, S.M.; Mahmud, J.A.; Fujita, M.; Fotopoulos, V. Reactive Oxygen Species and Antioxidant Defense in Plants under Abiotic Stress: Revisiting the Crucial Role of a Universal Defense Regulator. Antioxidants 2020, 9, 681. [CrossRef] [PubMed]

7. Noctor, G.; Mhamdi, A.; Foyer, C.H. The roles of reactive oxygen metabolism in drought: Not so cut and dried. Plant Physiol. 2014, 164, 1636-1648. [CrossRef]

8. Hasanuzzaman, M.; Bhuyan, M.; Anee, T.I.; Parvin, K.; Nahar, K.; Mahmud, J.A.; Fujita, M. Regulation of Ascorbate-Glutathione Pathway in Mitigating Oxidative Damage in Plants under Abiotic Stress. Antioxidants 2019, 8, 384. [CrossRef]

9. Asada, K. Production and scavenging of reactive oxygen species in chloroplasts and their functions. Plant Physiol. 2006, 141, 391-396. [CrossRef]

10. Foyer, C.H. Redox homeostasis: Opening up ascorbate transport. Nat. Plants 2015, 1, 14012. [CrossRef]

11. Debolt, S.; Melino, V.; Ford, C.M. Ascorbate as a biosynthetic precursor in plants. Ann. Bot. 2007, 99, 3-8. [CrossRef]

12. Cheng, F.; Lu, J.; Gao, M.; Shi, K.; Kong, Q.; Huang, Y.; Bie, Z. Redox Signaling and CBF-Responsive Pathway are Involved in Salicylic Acid-Improved Photosynthesis and Growth under Chilling Stress in Watermelon. Front. Plant Sci. 2016, 7, 1519. [CrossRef]

13. Han, Q.H.; Huang, B.; Ding, C.B.; Zhang, Z.W.; Chen, Y.E.; Hu, C.; Zhou, L.J.; Huang, Y.; Liao, J.Q.; Yuan, S.; et al. Effects of Melatonin on Anti-oxidative Systems and Photosystem II in Cold-Stressed Rice Seedlings. Front. Plant Sci. 2017, 8, 785. [CrossRef]

14. Wang, J.; Wu, B.; Yin, H.; Fan, Z.; Li, X.; Ni, S.; He, L.; Li, J. Overexpression of CaAPX Induces Orchestrated Reactive Oxygen Scavenging and Enhances Cold and Heat Tolerances in Tobacco. BioMed Res. Int. 2017, 2017, 4049534. 
15. Kwon, S.Y.; Choi, S.M.; Ahn, Y.O.; Lee, H.S.; Lee, H.B.; Park, Y.M.; Kwak, S.S. Enhanced stress-tolerance of transgenic tobacco plants expressing a human dehydroascorbate reductase gene. J. Plant Physiol. 2003, 160, 347-353. [CrossRef]

16. Green, M.R.; Sambrook, J. Constructing a Standard Curve for Real-Time Polymerase Chain Reaction (PCR) Experiments. Cold Spring Harb. Protoc. 2018, 2018, pdb-prot095026. [CrossRef] [PubMed]

17. Li, C.; Song, W.; Luo, Y.; Gao, S.; Zhao, J. The HuangZaoSi Maize Genome Provides Insights into Genomic Variation and Improvement History of Maize. Mol. Plant 2019, 12, 402-409. [CrossRef] [PubMed]

18. Raju, S.K.K.; Barnes, A.C.; Schnable, J.C.; Roston, R.L. Low-temperature tolerance in land plants: Are transcript and membrane responses conserved? Plant Sci. Int. J. Exp. Plant Biol. 2018, 276, 73-86.

19. Farmer, E.E.; Mueller, M.J. ROS-mediated lipid peroxidation and RES-activated signaling. Annu. Rev. Plant Biol. 2013, 64, 429-450. [CrossRef] [PubMed]

20. Ozturk, M.; Turkyilmaz Unal, B.; García-Caparrós, P.; Khursheed, A.; Gul, A.; Hasanuzzaman, M. Osmoregulation and its actions during the drought stress in plants. Physiol. Plant. 2021, 172, 1321-1335. [CrossRef] [PubMed]

21. Ishikawa, T.; Maruta, T.; Yoshimura, K.; Smirnoff, N. Biosynthesis and Regulation of Ascorbic Acid in Plants. In Antioxidants and Antioxidant Enzymes in Higher Plants; Gupta, D.K., Palma, J.M., Corpas, F.J., Eds.; Springer International Publishing: Cham, Switzerland, 2018; pp. 163-179.

22. Smirnoff, N. Ascorbic acid: Metabolism and functions of a multi-facetted molecule. Curr. Opin. Plant Biol. 2000, 3, $229-235$. [CrossRef]

23. Broad, R.C.; Bonneau, J.P.; Hellens, R.P.; Johnson, A.A.T. Manipulation of Ascorbate Biosynthetic, Recycling, and Regulatory Pathways for Improved Abiotic Stress Tolerance in Plants. Int. J. Mol. Sci. 2020, 21, 1790. [CrossRef]

24. Eastmond, P.J. MONODEHYROASCORBATE REDUCTASE4 is required for seed storage oil hydrolysis and postgerminative growth in Arabidopsis. Plant Cell 2007, 19, 1376-1387. [CrossRef] [PubMed]

25. Yoshida, S.; Tamaoki, M.; Shikano, T.; Nakajima, N.; Ogawa, D.; Ioki, M.; Aono, M.; Kubo, A.; Kamada, H.; Inoue, Y.; et al. Cytosolic dehydroascorbate reductase is important for ozone tolerance in Arabidopsis thaliana. Plant Cell Physiol. 2006, 47, 304-308. [CrossRef] [PubMed]

26. Ramazan, S.; Qazi, H.A.; Dar, Z.A.; John, R. Low temperature elicits differential biochemical and antioxidant responses in maize (Zea mays) genotypes with different susceptibility to low temperature stress. Physiol. Mol. Biol. Plants 2021, 27, 1395-1412. [CrossRef]

27. Yoshimura, K.; Nakane, T.; Kume, S.; Shiomi, Y.; Maruta, T.; Ishikawa, T.; Shigeoka, S. Transient expression analysis revealed the importance of VTC2 expression level in light/dark regulation of ascorbate biosynthesis in Arabidopsis. Biosci. Biotechnol. Biochem. 2014, 78, 60-66. [CrossRef] 